\title{
Review Article \\ Effectiveness and Safety of S-1-Based Therapy Compared with 5-Fluorouracil-Based Therapy for Advanced Colorectal Cancer: A Meta-Analysis
}

\author{
Jiaxiang Ye, Jiawei Chen, Lianying Ge, Aiqun Liu, and Shaozhang Zhou \\ Department of Medical Oncology, The Cancer Institute, Affiliated Tumor Hospital of Guangxi Medical University, Nanning, \\ Guangxi 530021, China
}

Correspondence should be addressed to Lianying Ge; gelianying2013@163.com

Received 8 April 2014; Accepted 4 November 2014; Published 30 November 2014

Academic Editor: Daiming Fan

Copyright (C) 2014 Jiaxiang Ye et al. This is an open access article distributed under the Creative Commons Attribution License, which permits unrestricted use, distribution, and reproduction in any medium, provided the original work is properly cited.

\begin{abstract}
Objectives. The aim of our study was to compare the efficacy and safety of S-1-based therapy (SBT) versus 5-fluorouracil-based therapy (FBT) for advanced colorectal cancer (ACRC). Methods. A meta-analysis of all eligible randomized controlled trials (RCTs) was performed using RevMan 5.1.0 software. Results. A total of 1625 patients from twelve RCTs including 820 patients in the SBT group and 805 patients in the FBT group were available for analysis. The meta-analysis of overall survival (hazards ratio $\mathrm{HR}=0.94$, $95 \% \mathrm{CI}=0.80-1.10)$, progression-free survival $(\mathrm{HR}=1.03,95 \% \mathrm{CI}=0.91-1.18)$, and overall response rate (odds ratio $\mathrm{OR}=1.23,95 \%$ $\mathrm{CI}=1.00-1.53)$ showed no statistical significance between SBT group and FBT group. The statistically significant differences in the meta-analysis indicated less incidence of graded 3-4 neutropenia $(\mathrm{OR}=0.49,95 \% \mathrm{CI}=0.35-0.68)$ and nausea/vomit $(\mathrm{OR}=0.41$, $95 \% \mathrm{CI}=0.23-0.72)$ in the SBT group, and there was no statistically significant difference in the incidence of grade 3-4 anemia, thrombocytopenia, leucopenia, diarrhea, and treatment-related deaths between two groups. Conclusions. SBT had similar efficacy and better safety than FBT and was an attractive alternative to FBT for patients of ACRC, but further investigations in different populations would be needed to confirm it.
\end{abstract}

\section{Introduction}

Despite advances in diagnosis and treatment, colorectal cancer remains the third leading cancer, with approximated $1,233,700$ new cases and 608,700 deaths worldwide each year [1]. For the patients with advanced colorectal cancer (ACRC), acquiring curative therapy by surgery or radiotherapy is complex; therefore, systemic chemotherapy is the main effective treatment, which can prolong survival and enhance life quality of patients [2].

For many years, traditional continuous-infusion 5-fluorouracil $(5-\mathrm{FU})$ in combination with leucovorin $(\mathrm{LV})$ has been the backbone of palliative therapy for ACRC [2], and the combination of 5-FU and LV with either oxaliplatin (FOLFOX) or irinotecan (FOLFIRI) has been recognized as standard first-line therapies for ACRC [3]. However, administration of the traditional 5-FU regimens is time-consuming, uncomfortable, and inconvenient for the patients, because continuous infusion requires an indwelling central venous catheter with the associated increased risk of infection and thrombosis and regular hospital visits.

As substitute of 5-FU, S-1 (Taiho Pharmaceutical Company, Tokyo, Japan), an oral fluoropyrimidine, is a combined form of three pharmacological compounds (tegafur, gimeracil [CDHP], and oteracil potassium [Oxo]) at a molar ratio of $1: 0.4: 1$. Tegafur is a prodrug that is mainly converted by liver enzyme cytochrome P450(CYP)2A6 to 5-FU, CDHP is an inhibitor of dihydropyrimidine dehydrogenase, which can prolong the half-life of 5-FU, and Oxo can reduce the toxic effects of 5-FU by inhibiting the phosphorylation of 5FU to fluorouridine monophosphate in the gastrointestinal tract [4]. S-1-based therapy (SBT) has found to have similar efficacy and safety to 5-fluorouracil-based therapy (FBT) in the treatment of advanced gastric cancer (AGC) [5] and has 
been approved for the treatment of patients with AGC in japan.

The question that whether SBT has similar efficacy and safety to FBT in the treatment of ACRC is well worth exploring and studying. Recently, there have been a series of trials comparing S-1 with 5-FU in mono or combined therapy for ACRC [6-17]. However, single study may not be powered sufficiently to comprehensively assess the efficacy and safety of them, and so far there still has been not a meta-analysis of SBT versus FBT for ACRC. Consequently, we performed the present meta-analysis of all eligible studies to compare both treatment approaches and to evaluate their clinical efficacy and safety for patients of ACRC.

\section{Methods}

2.1. Literature Search. We conducted a comprehensive search by examining the PubMed, Embase, and the Cochrane Library Database for randomized controlled trials (RCTs) from inception to November 15, 2013, using various combinations of different terms "colorectal cancer," "S-1," "5-FU," "randomized controlled trial," and their synonyms or similar words (the "appendix" showed the search strategy of Embase, and the search strategy was also referred in other electronic databases). In addition, all abstracts from the American Society of Clinical Oncology (ASCO) conferences from inception to 2013 were also searched for relevant RCTs, and references cited in the identified articles were searched manually. The search was done without restriction on language.

2.2. Inclusion and Exclusion Criteria. Inclusion and exclusion criteria were delineated before the commencement of the literature search. Eligible studies were included in this metaanalysis if they met all the following criteria: (1) that participants were the patients of histologically confirmed, advanced, recurrent, or metastatic colorectal cancer and did not have severe basic diseases, (2) that only RCT was considered, (3) trials comparing SBT with FBT: mono or combined therapy of S-1 versus 5-FU and not confused by additional drugs or interventions (i.e., the experimental and control arms had difference only by S-1 and 5-FU components in the combination therapy). Accordingly, studies meeting the following criteria were excluded: (1) crossover studies and (2) the studies about loses visit rate $>20 \%$.

2.3. Data Extraction. Essential data was carefully extracted from all eligible studies independently by two investigators (Jia-Xiang Ye, Lian-Ying Ge), and discrepancies were finally resolved by consensus between the two authors (Jia-Xiang Ye, Lian-Ying Ge). From each study, we collected information on the following items: the first author's name, published year, country/region of origin, study design, characteristics of participants, interventions, and outcomes. When there were some updated results about the same study, we extracted the updated results. For the included studies with only abstract, we also acquired relevant clinical trial information by ClinicalTrials.gov Database (http://www.clinicaltrials.gov/).
2.4. Quality Assessment for Included Studies. Two authors (Jia-Xiang Ye, Lian-Ying Ge) assessed the quality of the eligible studies independently, with disagreements solved by a third author (Shao-Zhang Zhou) until agreement was obtained. With the guidance of the Cochrane Collaboration's tool for assessing risk of bias of RCTs (5.1.0) [20], we considered the following criteria to appraise the RCTs: random sequence generation, allocation concealment, binding of participants and personnel, binding of outcome assessment, incomplete outcome data, selecting reporting, and other bias. In all cases, high risk, low risk, or unclear risk was used to evaluate the risk of bias, and if insufficient detail was reported of what happened in the study, the judgment would usually be unclear risk of bias.

2.5. Statistical Analysis. Statistical analysis of the hazards ratio (HR) and 95\% confidence interval (CI) for overall survival (OS) and progression-free survival (PFS), the odds ratio (OR) and 95\% CI for overall response rate (ORR), disease control rate (DCR), one- or two-year survival rate (SR), and grade 3-4 adverse events (AEs) were calculated using RevMan 5.1.0 software. ORR was defined as the sum of partial and complete response rates according to the Response Evaluation Criteria in Solid Tumors, and the DCR was the sum of ORR and stable disease rate [21]. A fixedeffect model was used first, the $Q$ test and $I^{2}$ statistic was performed to assess the heterogeneity, and $P \leq 0.1$ or $I^{2}>$ $50 \%$ was considered as heterogeneity between studies. If the heterogeneity existed, sensitivity analysis or random-effect model was applied. Visual inspection of asymmetry in funnel plots was used to estimate the potential publication bias [22]. In order to supplement the funnel plot, Begg's test [23] and Egger's test [24] methods were performed using Stata version 12.0 software (Stata Corporation, College Station, TX).

\section{Results}

3.1. Study and Patient Characteristics. The search strategy yielded 400 records. Of these, 79 duplicates were eliminated and 302 articles were excluded due to irrelevancy by reviewing their titles and abstracts. The remaining 19 records were obtained to further determine eligibility. We ruled out another five articles: two articles due to single arm trials $[25,26]$, one article due to pooled analysis [27], and two trials not comparing SBT with FBT $[28,29]$. So ten full texts $[6,7,9,11-17]$ and four abstracts $[8,10,18,19]$ were identified according to the inclusion criteria, of which the trial reported by Otsuji et al. [19] was the updated study of partial result of the trial reported by Ojima et al. [10], and the trial reported by Baba et al. [18] was the updated study of partial result of the trial reported by Muro et al. [9]. Thus, only twelve studies [6-17] assessing 1625 participants qualified to be included for this meta-analysis, as described in the flow chart (Figure 1). Table 1 displayed the characteristics of these twelve individual trials with respect to author (year), country, demographic data, duration, intervention, outcome measure, and study design.

3.2. Quality of Eligible Studies. All included studies undertook detailed assessments. All of the studies included the term 


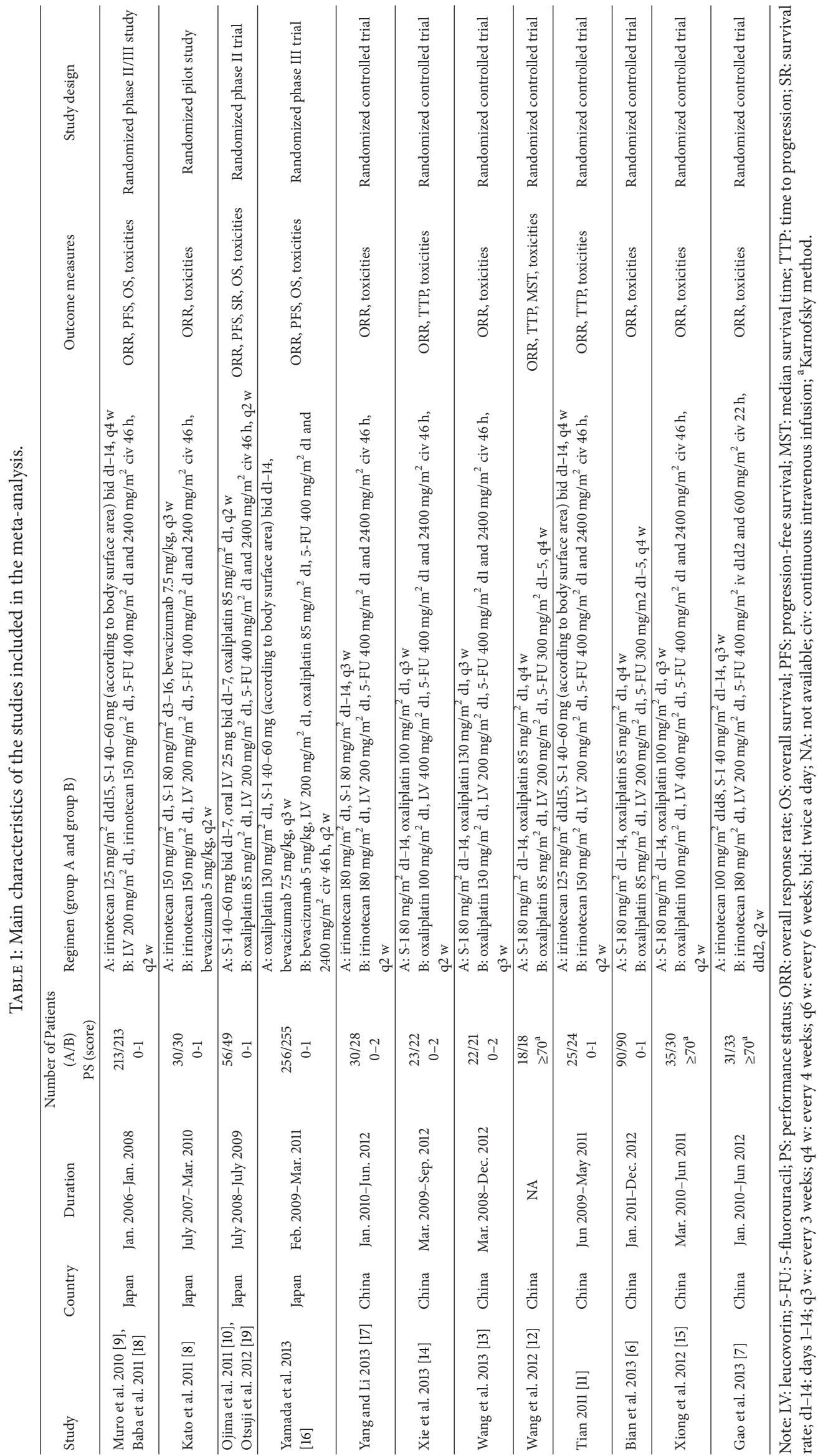




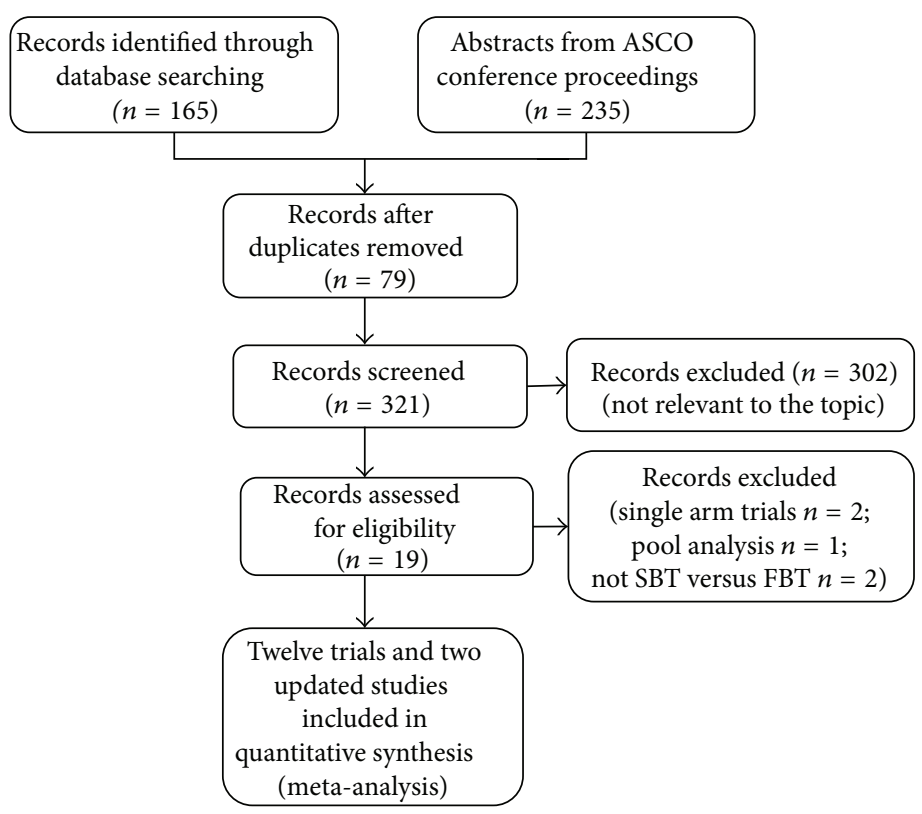

FIGURE 1: Flow chart displaying the process of study selection for the meta-analysis.

"random," but only three RCTs $[6,9,16]$ reported the methods of random sequence generation, and only two RCTs $[9,16]$ reported the allocation concealment in detail. Moreover, although the three full texts $[9,12,16]$ were open-label and other nine trials did not mention whether the blind method was adopted or not, these were unlikely to affect the quality assessment results. Two RCTs $[9,16]$ adequately described the missing data or missing reasons and took intention to treat analysis of all randomized patients. Eight RCTs [6, 7, 11-15, 17] reported complete outcome data. The ten RCTs had no other bias. Two trials $[8,10]$ were abstracts and included insufficient information regarding the outcome data, selective reporting and other bias (Figures 2 and 3).

3.3. Overall Survival. Three of the four trials provided OS data $[16,18,19]$. The pooled HR of OS showed no significant difference between SBT and FBT yielding HR of 0.94 (95\% CI 0.80-1.10) by using a fixed-effect model, and there was no significant heterogeneity across studies $\left(P=0.50, I^{2}=0 \%\right)$ (Figure 4).

3.4. One- or Two-Year Survival Rate. Three trials provided SR data $[9,10,16,19]$. The pooled OR of one- or two-year SR showed there was no significant difference between SBT and FBT (1-year SR: OR = 0.99, 95\% CI = 0.74-1.33; 2-year SR: OR $=1.01,95 \% \mathrm{CI}=0.76-1.35$ ) by using a fixed-effect model, with no heterogeneity across studies (1-year SR: $P=0.66, I^{2}=0 \%$; 2-year SR: $P=0.37, I^{2}=0 \%$ ) (Figure 5).

3.5. Progression-Free Survival. Three trials provided PFS data $[10,16,18]$. The pooled HR of PFS showed there was no significant difference between SBT and FBT (HR $=1.03,95 \%$ $\mathrm{CI}=0.91-1.18)$, and the pooled HR of PFS was performed by using fixed-effects model, with no heterogeneity $(P=0.69$, $I^{2}=0 \%$ ) (Figure 6).

3.6. Overall Response Rate or Disease Control Rate. All included studies provided the information on ORR [6-17]. As shown in Figure 7, the pooled OR of ORR in fixed-effect model was 1.23 (95\% CI: 1.00-1.53) with little evidence of heterogeneity $\left(P=0.62, I^{2}=0 \%\right)$, which indicated there was no significant difference between SBT group and FBT group. Eleven trials reported DCR data $[6-8,10-17]$, the meta-analysis of the pooled data demonstrated that DCR was not different between the two groups ( $\mathrm{OR}=1.37,95 \% \mathrm{CI}=$ 0.99-1.89), and there was no heterogeneity across the trials $\left(P=0.80, I^{2}=0 \%\right)$ (Figure 8$)$.

3.7. Safety. Results of graded 3 and 4 AEs analyses were shown in Table 2.

Neutropenia in hematologic toxicity: meta-analysis of four trials $[9-11,16]$ including 541 patients in the SBT group and 533 patients in the FBT group showed graded 3-4 neutropenia was less likely to happen in the SBT group (OR $=0.35,95 \% \mathrm{CI}=0.27-0.47)$, and yet there was significant heterogeneity across these trials $\left(P<0.1, I^{2}=69 \%\right)$. Sensitivity analysis suggested that the trial reported by Yamada et al. [16] was the main source of heterogeneity. After removing this study, the heterogeneity was eliminated $\left(P=0.73, I^{2}=\right.$ $0.0 \%)$, and the pooled result of the three trials applying fixedeffect model also showed that graded 3-4 neutropenia was significantly less likely to happen in patients of SBT than FBT $(\mathrm{OR}=0.49,95 \% \mathrm{CI}=0.35-0.68)$ (Figure 4(b)).

Leucopenia: meta-analysis of ten trials $[6,7,9,11-17]$ about graded 3-4 leucopenia, which included 734 patients in the SBT group and 726 patients in the FBT group, showed no significant difference between the two groups $(\mathrm{OR}=0.75$, 


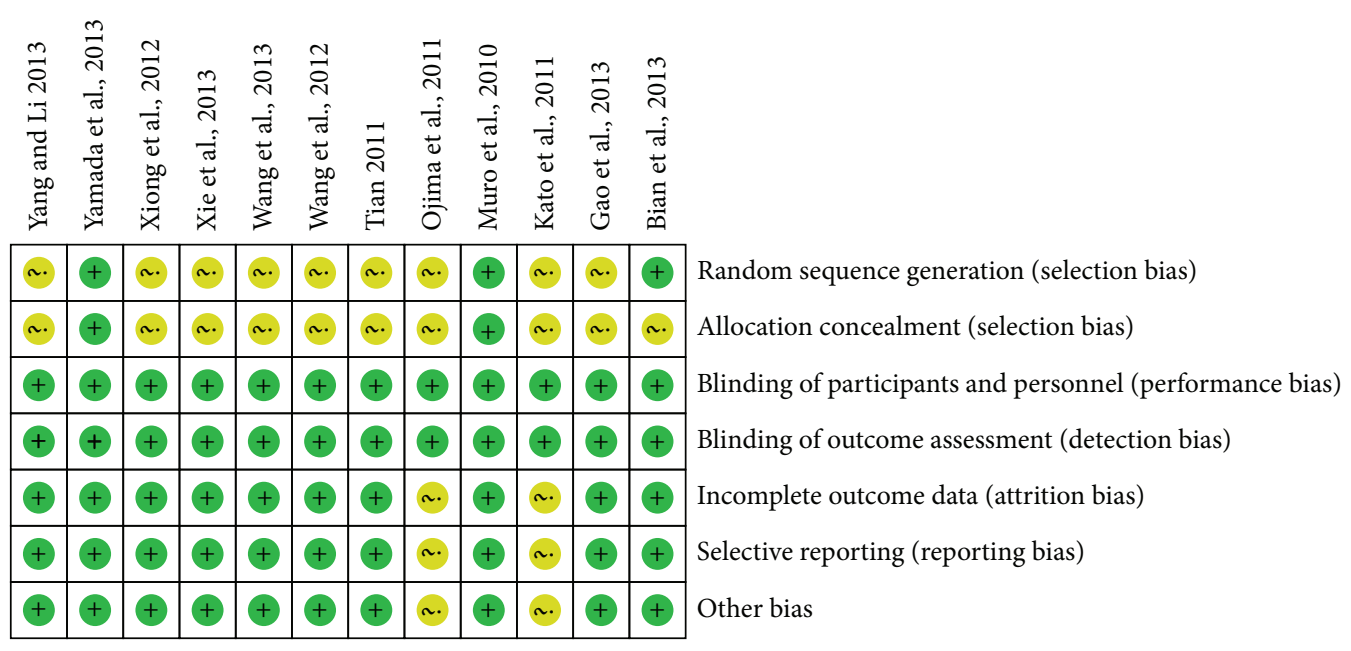

FIGURE 2: Risk of bias summary: review authors' judgements about each risk of bias item for each included study.

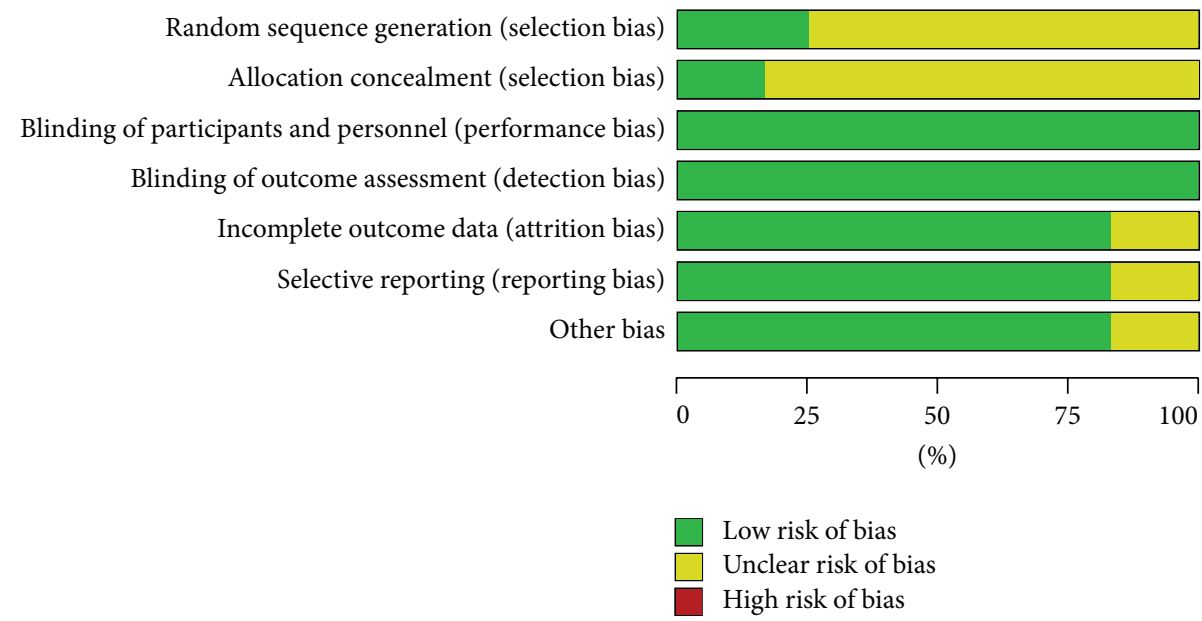

FIGURE 3: Risk of bias graph: review authors' judgements about each risk of bias item presented as percentages across all included studies.

95\% CI $=0.55-1.04)$, with no significant heterogeneity across studies $\left(P=0.28, I^{2}=18 \%\right)$.

Anemia: nine trials $[6,7,9,11,12,14-17]$ reported graded 3-4 anemia assessing 1417 participants (SBT, $n=712$; FBT, $n=$ $705)$, meta-analysis of the pooled data showed no significant difference between the two groups $(\mathrm{OR}=1.33,95 \% \mathrm{CI}=$ $0.83-2.15)$, and there was no significant heterogeneity across studies $\left(P=0.40, I^{2}=3 \%\right)$.

Thrombocytopenia: the pooled OR of graded 3-4 thrombocytopenia of nine trials $[6,7,9,12-17]$ assessing 1411 participants (SBT, $n=709$; FBT, $n=702$ ) showed no significant difference between the two groups (OR $=1.05,95 \%$ $\mathrm{CI}=0.51-2.15)$, and there was no significant heterogeneity across studies $\left(P=0.28, I^{2}=21 \%\right)$.

Diarrhea in nonhematologic toxicity: all included studies provided the information on grade 3-4 diarrheas. Metaanalysis of twelve trials showed a significant heterogeneity across the trials $\left(P<0.10, I^{2}=64 \%\right)$. Sensitivity analysis did not find the main source of heterogeneity. So meta-analysis of twelve trials assessing 1625 participants (SBT, $n=820$; FBT, $n=805)$ in random-effect model showed no significant difference between the two groups $(\mathrm{OR}=1.25,95 \% \mathrm{CI}=0.58$ 2.69).

Nausea/vomit: meta-analysis of ten trials [6-9, 12-17] assessing 1471 participants (SBT, $n=739$; FBT, $n=732$ ) in fixed-effect model showed no significant difference between the two groups $(\mathrm{OR}=0.41,95 \% \mathrm{CI}=0.23-0.72)$, with no heterogeneity $\left(P=0.56, I^{2}=0 \%\right)$.

Stomatitis: nine trials $[6-9,12,13,15-17]$ reported graded 3-4 stomatitis assessing 1426 participants (SBT, $n=716$; FBT, $n=710$ ), meta-analysis of the pooled data showed no significant difference between the two groups (OR $=2.21,95 \%$ $\mathrm{CI}=0.83-5.88)$, and there was no significant heterogeneity across studies $\left(P=0.24, I^{2}=28 \%\right)$.

Treatment-related death (TRD): data on the TRD were available for five trials $[8,9,11,16,17]$ including 1087 participants (SBT, $n=545$; FBT, $n=542$ ) in the metaanalysis. The pooled OR of five trials showed TRD was not 


\begin{tabular}{|c|c|c|c|c|c|c|c|}
\hline \multirow{2}{*}{$\frac{\text { Study or subgroup }}{\text { Muro et al., } 2010}$} & & \multirow{2}{*}{$\begin{array}{c}\text { Hazard ratio } \\
\text { IV, fixed, 95\% CI } \\
0.90[0.73,1.12]\end{array}$} & \multicolumn{5}{|c|}{$\begin{array}{c}\text { Hazard ratio } \\
\text { IV, fixed, } 95 \% \text { CI }\end{array}$} \\
\hline & $56.5 \%$ & & & & & & \\
\hline Ojima et al., 2011 & $9.0 \%$ & $0.76[0.45,1.30]$ & & & & & \\
\hline Yamada et al., 2013 & $34.5 \%$ & $1.05[0.80,1.38]$ & & & & & \\
\hline Total $(95 \%$ CI $)$ & $100.0 \%$ & $0.94[0.80,1.10]$ & & & $\lambda$ & & \\
\hline \multicolumn{3}{|c|}{ Heterogeneity: $\chi^{2}=1.39, \mathrm{df}=2(P=0.50) ; I^{2}=0 \%$} & $\Gamma$ & $T$ & 1 & $T$ & \\
\hline \multirow{2}{*}{\multicolumn{3}{|c|}{ Test for overall effect: $Z=0.82(P=0.41)$}} & 0.01 & 0.1 & 1 & 10 & 100 \\
\hline & & & \multicolumn{3}{|c|}{ Favours [S-1] } & urs & $\mathrm{FU}]$ \\
\hline
\end{tabular}

FIGURE 4: Forest plot of hazard ratio of overall survival.

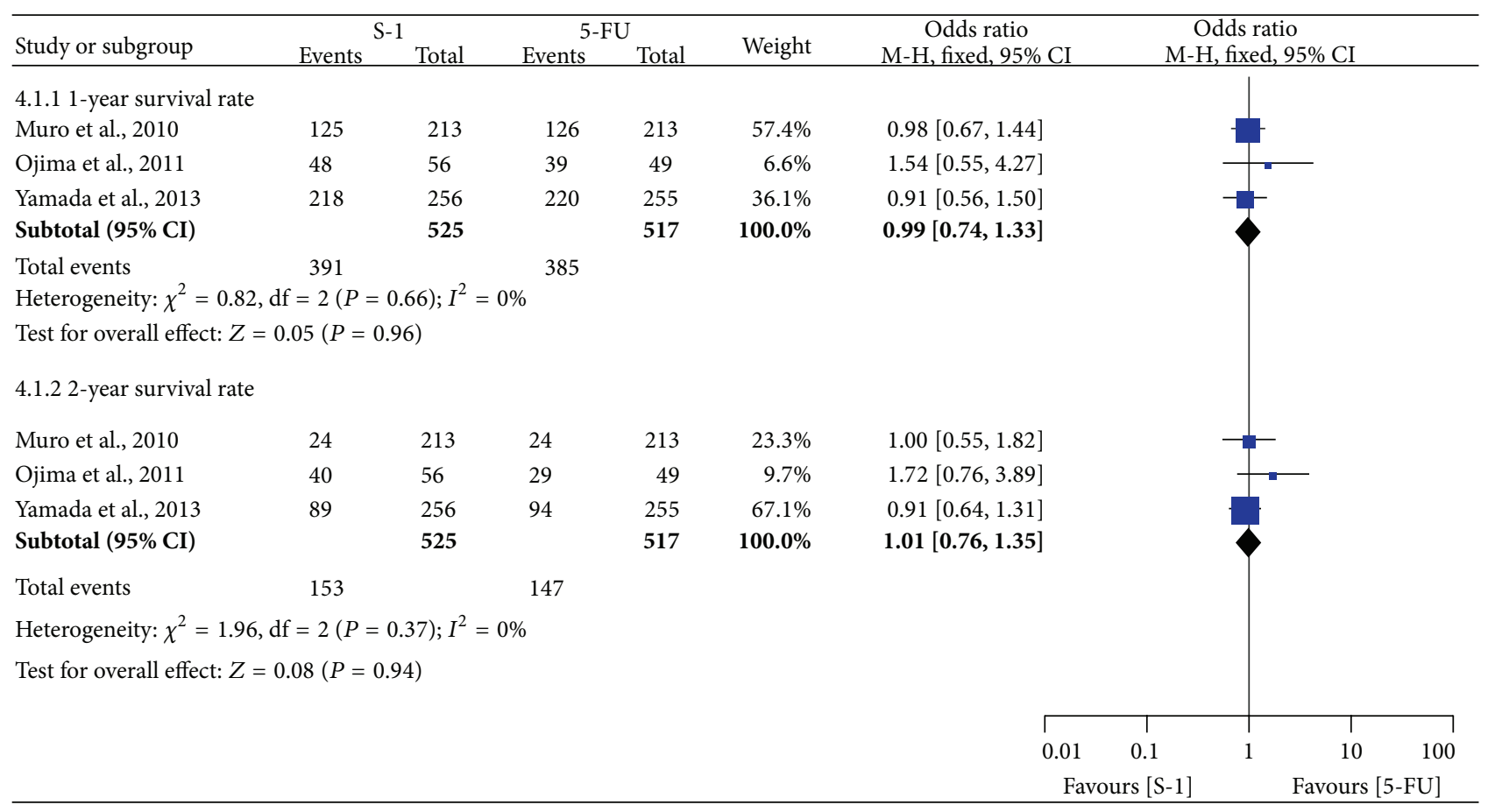

FIgURE 5: Forest plot of odds ratio of one- or two-year survival rate.

significantly different between the two groups $(\mathrm{OR}=0.72$, $95 \% \mathrm{CI}=0.24-2.19)$, and there was no heterogeneity among the studies $\left(P=0.71, I^{2}=0 \%\right)$.

3.8. Publication Bias. The visual inspection of the funnel plots suggested a roughly symmetrical distribution for the study (Figure 9), which showed no evidence of publication bias. Moreover, according to Begg's test $(P=0.49)$ and Egger's test $(P=0.40)$, publication bias was also not found.

\section{Discussion}

To our knowledge, this was the first meta-analysis to evaluate the efficacy and safety of SBT versus FBT for ACRC. A total of 1625 patients from twelve RCTs including 820 patients in the SBT group and 805 patients in the FBT group were analyzed. With respect to ORR, DCR, and one- or two-year SR, our meta-analysis showed no significant difference between SBT and FBT group, which suggested that SBT was noninferior to FBT for the patients of ACRC. And the pooled analysis showed that SBT had similar PFS and OS to FBT as well. All these results indicated SBT had similar efficacy to FBT for patients of ACRC.

For safety profile, Our meta-analysis showed grade 34 toxicity such as anemia, leucopenia, thrombocytopenia, diarrhea, and TRD was similar between two groups, but the graded 3-4 neutropenia $(\mathrm{OR}=0.49$, 95\% CI $=0.35-$ $0.68)$ and nausea/vomit $(\mathrm{OR}=0.41,95 \% \mathrm{CI}=0.23-0.72)$ were less likely to happen in the SBT group than in the FBT group. Thus, compared with FBT, SBT could reduce some toxicity for patients of ACRC. Owing to significant heterogeneity in grade 3-4 neutropenia, sensitivity analysis was performed to find the contributors of heterogeneity, the trial reported by Yamada et al. [16], and the main source of heterogeneity might be attributable to the different therapy regimens; for instance, the therapy regimen of the trial reported by Yamada et al. had a biological targeted drug (bevacizumab), but the therapy regimens of the other studies of meta-analysis did not include bevacizumab. Regarding grade 3-4 diarrhea, a sensitivity analysis was also performed, 


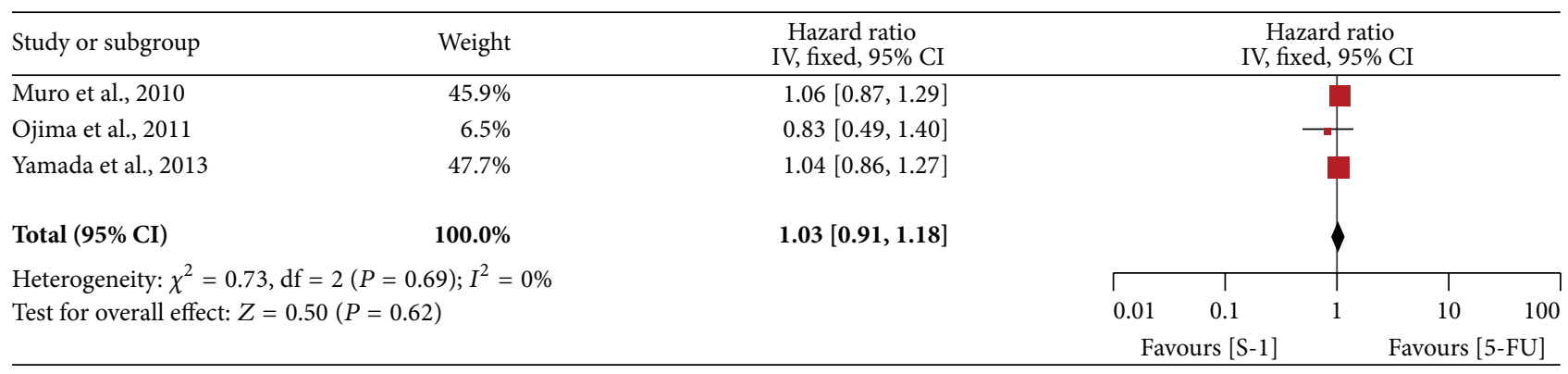

FIGURE 6: Forest plot of hazard ratio of progression-free survival.

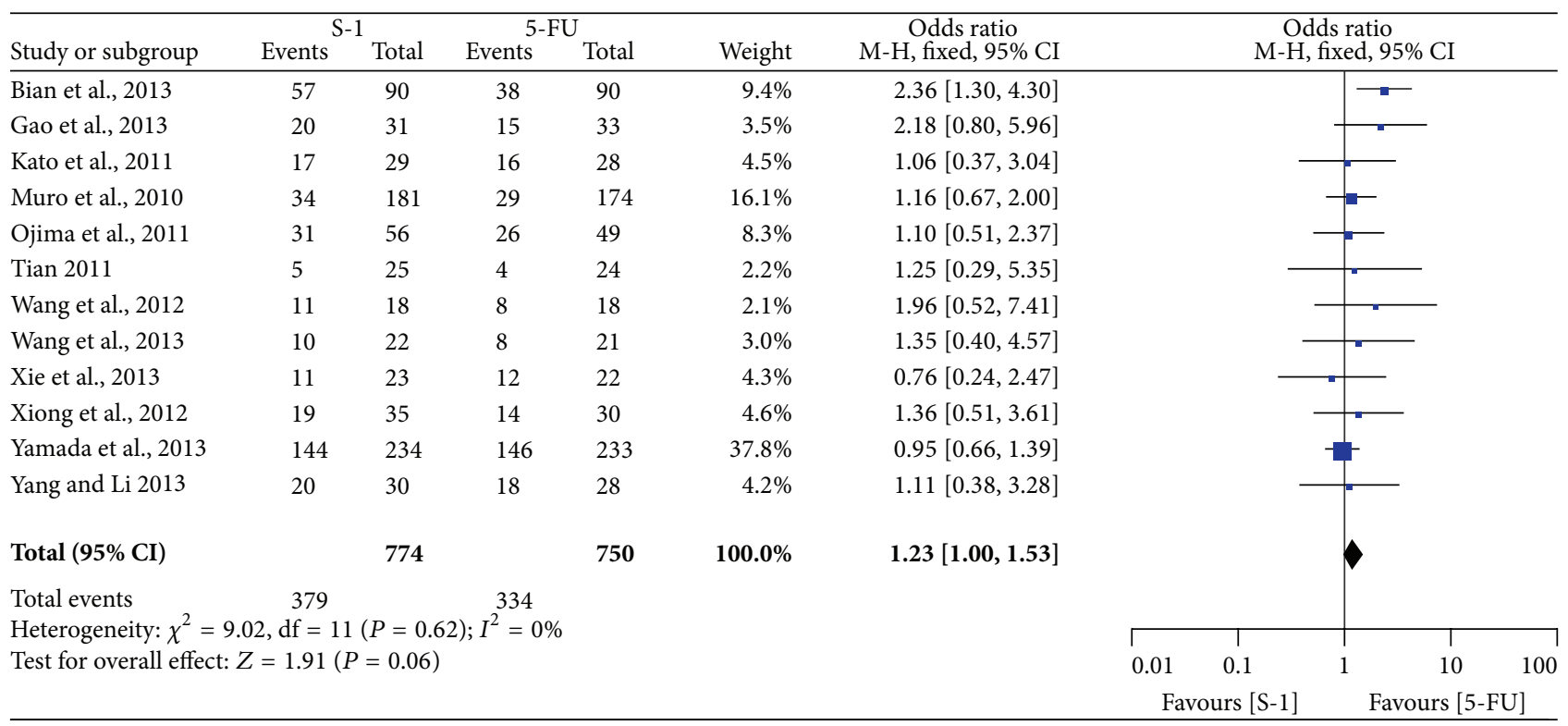

FIGURE 7: Forest plot of odds ratio of overall response rate.

\begin{tabular}{|c|c|c|c|c|c|c|c|c|c|c|}
\hline \multirow{2}{*}{$\frac{\text { Study or subgroup }}{\text { Bian et al., } 2013}$} & \multicolumn{2}{|c|}{ S-1 } & \multicolumn{2}{|c|}{ 5-FU } & Weight & $\begin{array}{c}\text { Odds ratio } \\
\text { M-H, fixed, 95\% CI }\end{array}$ & \multicolumn{4}{|c|}{$\begin{array}{c}\text { Odds ratio } \\
\text { M-H, fixed, 95\% CI }\end{array}$} \\
\hline & 75 & 90 & 66 & 90 & $17.4 \%$ & $1.82[0.88,3.75]$ & & & 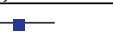 & \\
\hline Gao et al., 2013 & 26 & 31 & 24 & 33 & $5.9 \%$ & $1.95[0.57,6.64]$ & & & 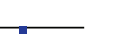 & \\
\hline Kato et al., 2011 & 28 & 29 & 26 & 28 & $1.4 \%$ & $2.15[0.18,25.19]$ & & & & \\
\hline Ojima et al., 2011 & 52 & 56 & 42 & 49 & $5.1 \%$ & $2.17[0.59,7.90]$ & & & & \\
\hline Tian 2011 & 19 & 25 & 16 & 24 & $6.2 \%$ & $1.58[0.45,5.53]$ & & & & \\
\hline Wang et al., 2012 & 15 & 18 & 12 & 18 & $3.2 \%$ & $2.50[0.51,12.14]$ & & & & \\
\hline Wang et al., 2013 & 17 & 22 & 15 & 21 & $5.5 \%$ & $1.36[0.34,5.38]$ & & & & \\
\hline Xie et al., 2013 & 16 & 23 & 19 & 22 & $9.4 \%$ & $0.36[0.08,1.63]$ & & & & \\
\hline Xiong et al., 2012 & 29 & 35 & 23 & 30 & $6.7 \%$ & $1.47[0.43,4.98]$ & & &. & \\
\hline Yamada et al., 2013 & 209 & 234 & 208 & 233 & $35.2 \%$ & $1.00[0.56,1.81]$ & & & & \\
\hline Yang and Li 2013 & 27 & 30 & 24 & 28 & $3.9 \%$ & $1.50[0.30,7.39]$ & & & & \\
\hline Total $(95 \% \mathrm{CI})$ & & 593 & & 576 & $100.0 \%$ & $1.37[0.99,1.89]$ & & & & \\
\hline Total events & 513 & & 475 & & & & & & & \\
\hline \multicolumn{7}{|c|}{ Heterogeneity: $\chi^{2}=6.23, \mathrm{df}=2(P=0.80) ; I^{2}=0 \%$} & & & $T$ & \\
\hline \multicolumn{7}{|c|}{ Test for overall effect: $Z=1.92(P=0.06)$} & $0.01 \quad 0.1$ & & 10 & 100 \\
\hline & & & & & & & \multicolumn{4}{|c|}{ Favours $[S-1]$} \\
\hline
\end{tabular}

FIGURE 8: Forest plot of odds ratio of disease control rate. 
TABLE 2: Outcome of toxicity meta-analysis comparing S-1 versus 5-FU in advanced colorectal cancer.

\begin{tabular}{lccccccrr}
\hline \multirow{2}{*}{ Toxicity } & \multirow{2}{*}{ Trials } & \multirow{2}{*}{ SBT } & \multirow{2}{*}{ FBT } & \multicolumn{2}{c}{ Heterogeneity } & \multirow{2}{*}{ OR (95\% CI) } & \multirow{2}{*}{ Model } & \multirow{2}{*}{$P$ value } \\
& & & & & $P$ value & $I^{2}(\%)$ & & \\
\hline Grade 3-4 neutropenia & 3 & $96 / 291$ & $143 / 284$ & 0.73 & 0 & $0.49[0.35,0.68]$ & Fixed & $<0.01$ \\
Grade 3-4 leucopenia & 10 & $79 / 734$ & $100 / 726$ & 0.28 & 18 & $0.75[0.55,1.04]$ & Fixed & 0.08 \\
Grade 3-4 anemia & 9 & $41 / 712$ & $31 / 705$ & 0.40 & 3 & $1.33[0.83,2.15]$ & Fixed & 0.24 \\
Grade 3-4 thrombocytopenia & 9 & $15 / 709$ & $14 / 702$ & 0.28 & 21 & $1.05[0.51,2.15]$ & Fixed & 0.89 \\
Grade 3-4 diarrhea & 12 & $92 / 820$ & $49 / 805$ & $<0.1$ & 64 & $1.25[0.58,2.69]$ & Random & 0.57 \\
Grade 3-4 nausea/vomit & 10 & $15 / 739$ & $38 / 732$ & 0.56 & 0 & $0.41[0.23,0.72]$ & Fixed & $<0.01$ \\
Grade 3-4 stomatitis & 9 & $11 / 716$ & $4 / 710$ & 0.24 & 28 & $2.21[0.83,5.88]$ & Fixed & 0.11 \\
Treatment-related death & 5 & $4 / 545$ & $6 / 542$ & 0.71 & 0 & $0.72[0.24,2.19]$ & Fixed & 0.57 \\
\hline
\end{tabular}

Notes: NA: not available; OR: odds ratio; CI: confidence interval; FBT: 5-fluorouracil-based therapy; SBT: S-1-based therapy.

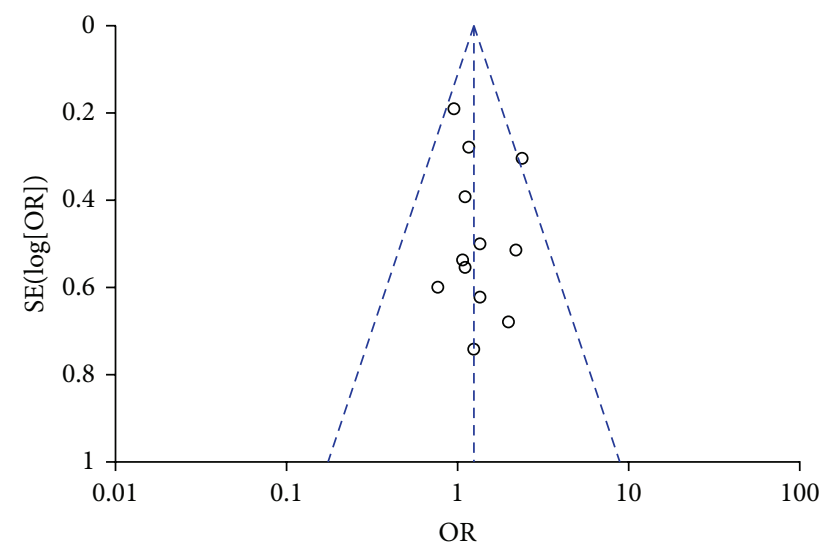

FIGURE 9: Funnel plot.

but the factors contributing to the heterogeneity could not be identified; these might be associated with variations in age, performance status of patients, dose, and the regimen of therapy between the trials. Thus, a random-effect model was applied to compensate for this.

Capecitabine is another oral fluorouracil-derivative drug designed to simulate a continuous intravenous infusion of 5-FU [30], whose efficacy and safety had been found to be at least equivalent to that of 5-FU for ACRC by many studies [31]. And yet, in 2012 the phase 3 trial reported by Hong et al. showed the noninferiority of oxaliplatin and S-1(SOX) versus oxaliplatin and capecitabine (XELOX) for ACRC, with median PFS of 8.5 months in the SOX group and 6.7 months in the XELOX group (HR $=0.79,95 \%$ CI $0.60-$ 1.04), and manageable toxicities in both groups [28]. Later the results of a randomized phase II study also suggested that both SOX and XELOX regimens were active and were well tolerated regimens in patients with ACRC [29]. These studies suggested indirectly that S-1-based chemotherapy (SBCT) could be regarded as an alternate chemotherapy strategy to 5-FU-based chemotherapy (FBCT) for the patients of ACRC.

Recently 5-FU-based chemotherapy plus bevacizumab $(\mathrm{FBCT}+\mathrm{Bev})$ has been widely used for first-line treatment of ACRC [32, 33], but S-1-based chemotherapy plus bevacizumab (SBCT $+\mathrm{Bev})$ is still at the research stage. In 2013,
Yamada et al. performed a randomised phase 3 trial, showing that SBCT + Bev was noninferior to FBCT + Bev in the firstline treatment for ACRC, with median PFS of 11.7 months in SBT group and 11.5 months in FBT group, and AEs in both groups were tolerable [16]. Moreover, the median PFS of SBCT + Bev was about 2 months longer than that of similar SBCT without bevacizumab in the treatment of patients with similar characteristics in the phase III trial reported by Hong et al. [28]. What is more, SBCT + Bev did not require a long infusion process and reduced the inconvenience for patients. Therefore, compared with FBCT + Bev, SBCT + Bev provided a great advantage and was a promising regimen for patients of ACRC.

Of note, S-1 had different optimal doses and safety among patients in different regions and populations. Since the gene polymorphic variants of liver enzyme CYP2A6 converting tegafur to 5-FU were less frequent in the Caucasians than in East Asians, the tolerable dose of S-1 might be lower for Caucasian patients than for patients in East Asia; in other words, toxic effects were more common in Caucasian patients exposed to the same dose of S-1 [34, 35]. Besides, variation in creatinine clearance was associated with S-1 toxicity as well, which meant dose adjustment should be considered when S-1 was used for patients with compromised renal function [16]. Thus, the use of different dose of S- 1 should be clarified for suitable population.

There were several limitations in this meta-analysis. First, since all the studies included in this analysis were from East Asia, the results needed confirmation in other countries. Second, the quality of the studies was not considered to be high, only three RCTs $[6,9,16]$ reported the methods of random sequence generation, and two RCTs $[9,16]$ reported the allocation concealment in detail. More RCTs with improved methodological quality should be provided to update this study. Third, two studies in the meta-analysis were abstracts $[8,10]$, whose incomplete information might potentially limit estimate of SBT effects. Moreover, information of each individual patient for each trial was not obtained to make comprehensive analysis. Last, there was one heterogeneous result about AEs.

In summary, our meta-analysis indicated that SBT had similar efficacy and better safety than FBT for patients of ACRC. Given its advantages of simplicity and convenience 
to administer, SBT would be an attractive alternative to FBT for patients of ACRC, especially for outpatients. Owing to the variation of S-1 tolerance in different regions and populations, further high-quality RCTs and different population studies in future would be needed to confirm it.

\section{Appendix}

EMBASE search terms:

(1) "colorectal": ab, ti OR "rectal": ab, ti OR "colon": ab, ti OR “intestinal”: ab, ti OR "colorectal carcinoma"/exp OR "colorectal tumor"/exp;

(2) "tegafur gimeracil oteracil potassium": ab, ti OR "S1": ab, ti OR “TS-1": ab, ti OR “gimeracil plus oteracil potassium plus tegafur"/exp;

(3) "fluorouracil": ab, ti OR "5-FU": ab, ti OR "fluorouracil"/exp;

(4) "trial": ab, ti OR "randomized": ab, ti OR "randomly": ab, ti OR "random": ab, ti OR "groups": ab, ti OR "placebo": ab, ti OR "randomized controlled trial”/exp OR "randomized controlled trial (topic)"/exp;

(5) \#1 AND \#2 AND \#3 AND \#4.

\section{Conflict of Interests}

The authors declare that they have no conflict of interests.

\section{Authors' Contribution}

Jiaxiang Ye and Jiawei Chen contributed equally to this work.

\section{References}

[1] A. Jemal, F. Bray, M. M. Center, J. Ferlay, E. Ward, and D. Forman, "Global cancer statistics," CA: A Cancer Journal for Clinicians, vol. 61, no. 2, pp. 69-90, 2011.

[2] J. A. Meyerhardt and R. J. Mayer, "Systemic therapy for colorectal cancer," The New England Journal of Medicine, vol. 352, no. 5, pp. 476-487, 2005.

[3] B. H. O'Neil and R. M. Goldberg, "Innovations in chemotherapy for metastatic colorectal cancer: an update of recent clinical trials," The Oncologist, vol. 13, no. 10, pp. 1074-1083, 2008.

[4] T. Shirasaka, Y. Shimamato, H. Ohshimo et al., "Development of a novel form of an oral 5-fluorouracil derivative (S-1) directed to the potentiation of the tumor selective cytotoxicity of 5-fluorouracil by two biochemical modulators," Anti-Cancer Drugs, vol. 7, no. 5, pp. 548-557, 1996.

[5] J. Huang, Y. Cao, L. Wu, C. Liao, Y. He, and F. Gao, "S-1-based therapy versus 5-FU-based therapy in advanced gastric cancer: a meta-analysis," Medical Oncology, vol. 28, no. 4, pp. 1004-1011, 2011.

[6] C. J. Bian, J. Zhu, and Q. Sun, "Clinical study of oxaliplatin plus S-1 in the treatment of advanced colorectal cancer," Chinese Journal of Primary Medicine and Pharmacy, vol. 20, no. 20, pp. 3133-3134, 2013.

[7] Y. L. Gao, Y. M. Han, and J. F. Hou, "Clinical effect observation of S-1 combined with irinotecan for patients with advanced colorectal carcinoma," Medical Innovation of China, vol. 10, no. 16, pp. 17-19, 2013.

[8] S. Kato, H. Andoh, M. Gamoh et al., "A randomized pilot study comparing safety and efficacy of irinotecan plus S-1 (IRIS) plus bevacizumab (BV) and modified (m) FOLFIRI plus BV in patients (pts) with metastatic colorectal cancer (mCRC): first report of T-CORE0702," Journal of Clinical Oncology, vol. 29, no. 4, p. 496, 2011.

[9] K. Muro, N. Boku, Y. Shimada et al., "Irinotecan plus S-1 (IRIS) versus fluorouracil and folinic acid plus irinotecan (FOLFIRI) as second-line chemotherapy for metastatic colorectal cancer: a randomised phase $2 / 3$ non-inferiority study (FIRIS study)," The Lancet Oncology, vol. 11, no. 9, pp. 853-860, 2010.

[10] H. Ojima, K. Yamazaki, H. Kuwano et al., "Randomized phase II study of S-1, oral leucovorin, and oxaliplatin combination therapy (SOL) versus mFOLFOX6 in patients with untreated metastatic colorectal cancer (mCRC)," European Journal of Cancer, vol. 47, supplement 1, pp. S427-S428, 2011.

[11] S. M. Tian, "S-1 plus irinotecan for second-line treatment of patients with metastatic colorectal cancer," Journal of Qiqihar Medical College, vol. 32, no. 16, pp. 2580-2582, 2011.

[12] X. L. Wang, J. R. Peng, and X. H. Wang, "Clinical study of S-1 capsule combined with oxaliplatin in the treatment of patients with advanced colorectal carcinoma," Journal of Modern Oncology, vol. 20, no. 10, pp. 2104-2106, 2012.

[13] Y. Wang, Y. S. Zhang, and J. Wang, "Clinical study of S-1 capsule plus oxaliplatin compared with FOLFOX6 in the treatment of patients with advanced colorectal carcinoma," Anhui Medical Journal, vol. 34, no. 9, pp. 1309-1311, 2013.

[14] M. Xie, F. Ma, and H. Y. Li, "Clinical study of S-1 combined with oxaliplatin in the treatment of patients with advanced colorectal carcinoma," Chinese Journal of Oncology Prevention and Treatment, vol. 5, no. 2, pp. 159-161, 2013.

[15] R. H. Xiong, Q. Ren, and X. R. Tian, "Clinical research of advanced colon carcinoma patients treated with S-1 combined with oxaliplatin," Chinese Journal of Medicinal Guide, vol. 14, no. 7, pp. 1208-1210, 2012.

[16] Y. Yamada, D. Takahari, H. Matsumoto et al., "Leucovorin, fluorouracil, and oxaliplatin plus bevacizumab versus S-1 and oxaliplatin plus bevacizumab in patients with metastatic colorectal cancer (SOFT): an open-label, non-inferiority, randomised phase 3 trial," The Lancet Oncology, vol. 14, no. 13, pp. 1278-1286, 2013.

[17] K. Yang and X. F. Li, "Clinical study of irinotecan combined with tegafur compared with irinotecan combined with 5-fluorouracil in treatment of advanced rectal cancer," Chinese Journal of Medicinal Guide, vol. 15, no. 7, pp. 1278-1279, 2013.

[18] H. Baba, K. Muro, H. Yasui et al., "Updated results of the FIRIS study: a phase II/III trial of 5-FU/l-leucovorin/irinotecan (FOLFIRI) versus irinotecan/S-1 (IRIS) as second-line chemotherapy for metastatic colorectal cancer (mCRC)," Journal of Clinical Oncology, vol. 29, no. 15, p. 3562, 2011.

[19] T. Otsuji, K. Yamazaki, H. Ojima et al., "Updated survival results of the randomized phase II study of S-1, oral leucovorin, and oxaliplatin combination therapy (SOL) versus mFOLFOX6 in patients with untreated metastatic colorectal cancer (mCRC)," Journal of Clinical Oncology, vol. 30, no. 4, p. 586, 2012.

[20] J. P. T. Higgins and S. Green, Cochrane Handbook for Systematic Reviews of Interventions Version 5.1.0, The Cochrane Collaboration, 2011, http://handbook.cochrane.org/.

[21] P. Therasse, S. G. Arbuck, E. A. Eisenhauer et al., "New guidelines to evaluate the response to treatment in solid tumors," 
Journal of the National Cancer Institute, vol. 92, no. 3, pp. 205216, 2000.

[22] D. A. Bennett, N. K. Latham, C. Stretton, and C. S. Anderson, "Capture-recapture is a potentially useful method for assessing publication bias," Journal of Clinical Epidemiology, vol. 57, no. 4, pp. 349-357, 2004.

[23] C. B. Begg and M. Mazumdar, "Operating characteristics of a rank correlation test for publication bias," Biometrics, vol. 50, no. 4, pp. 1088-1101, 1994.

[24] M. Egger, G. D. Smith, M. Schneider, and C. Minder, "Bias in meta-analysis detected by a simple, graphical test," British Medical Journal, vol. 315, no. 7109, pp. 629-634, 1997.

[25] Y. Komatsu, S. Yuki, and S. Sogabe, "Phase II trial of combined chemotherapy with irinotecan, $S-1$, and bevacizumab in patients with metastatic colorectal cancer," Annals of Oncology, vol. 21, supplement 8, p. viiil97, 2010.

[26] Y. Choi, T. Kim, S. Lee et al., "A phase I/II study of combination therapy of S-1 and irinotecan in patients with previously untreated metastatic or recurrent colorectal cancer," Journal of Clinical Oncology, vol. 27, no. 15, Article ID e15023, 2009.

[27] A. Goto, Y. Yamada, S. Iwasa et al., "S-1 and irinotecan versus 5-fluorouracil and leucovorin plus oxaliplatin with or without bevacizumab in metastatic colorectal cancer: a pooled analysis of 4 phase II studies," European Journal of Cancer, vol. 49, supplement 2, pp. S551-S597, 2013.

[28] Y. S. Hong, Y. S. Park, H. Y. Lim et al., "S-1 plus oxaliplatin versus capecitabine plus oxaliplatin for first-line treatment of patients with metastatic colorectal cancer: a randomised, noninferiority phase 3 trial," The Lancet Oncology, vol. 13, no. 11, pp. 1125-1132, 2012.

[29] D. Y. Zang, I. J. Chung, H. S. Oh, K. U. Park, K. H. Lee, and B. Han, "Randomized phase II study of oxaliplatin and S-1 (OS) versus oxaliplatin and capecitabine (XELOX) in patients with advanced or recurrent colorectal cancer," Journal of Clinical Oncology, vol. 30, no. 4, p. 595, 2012.

[30] M. Miwa, M. Ura, M. Nishida et al., "Design of a novel oral fluoropyrimidine carbamate, capecitabine, which generates 5 fluorouracil selectively in tumours by enzymes concentrated in human liver and cancer tissue," European Journal of Cancer, vol. 34, no. 8, pp. 1274-1281, 1998.

[31] W. Ling, J. Fan, Y. Ma, and H. Wang, "Capecitabine-based chemotherapy for metastatic colorectal cancer," Journal of Cancer Research and Clinical Oncology, vol. 137, no. 6, pp. 927938, 2011.

[32] H. Hurwitz, L. Fehrenbacher, W. Novotny et al., "Bevacizumab plus irinotecan, fluorouracil, and leucovorin for metastatic colorectal cancer," The New England Journal of Medicine, vol. 350, no. 23, pp. 2335-2342, 2004.

[33] L. B. Saltz, S. Clarke, E. Díaz-Rubio et al., "Bevacizumab in combination with oxaliplatin-based chemotherapy as first-line therapy in metastatic colorectal cancer: a randomized phase III study," Journal of Clinical Oncology, vol. 26, no. 12, pp. 20132019, 2008.

[34] J. A. Ajani, J. Faust, K. Ikeda et al., "Phase I pharmacokinetic study of S-1 plus cisplatin in patients with advanced gastric carcinoma," Journal of Clinical Oncology, vol. 23, no. 28, pp. 6957-6965, 2005.

[35] B. Chuah, B.-C. Goh, S.-C. Lee et al., "Comparison of the pharmacokinetics and pharmacodynamics of S-1 between Caucasian and East Asian patients," Cancer Science, vol. 102, no. 2, pp. 478483, 2011. 


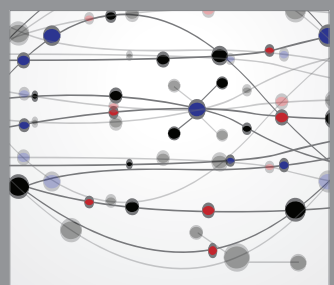

The Scientific World Journal
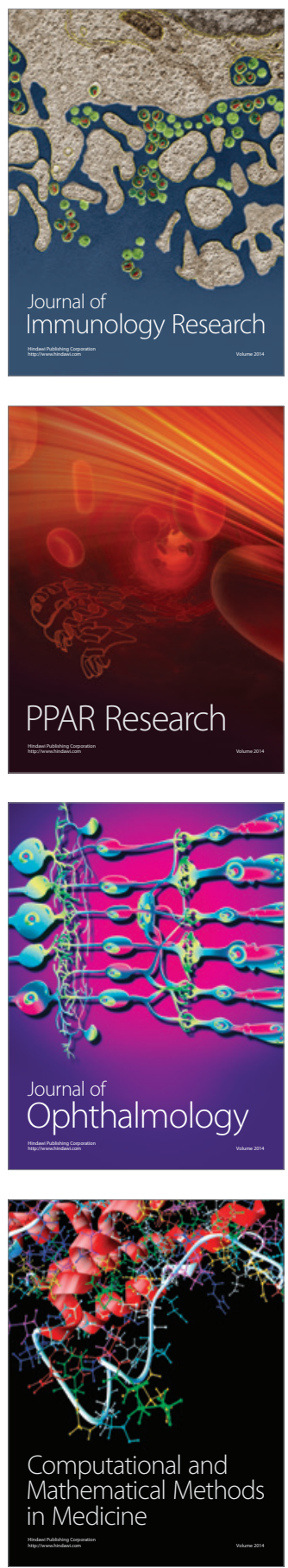

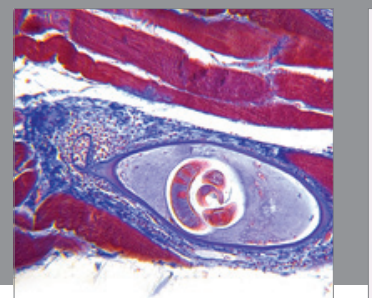

Gastroenterology

Research and Practice
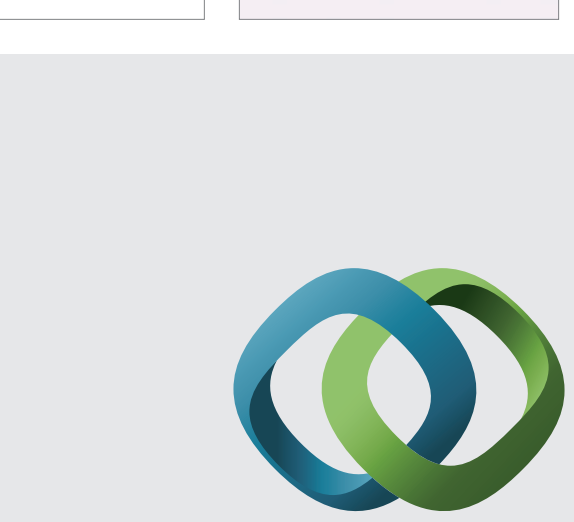

\section{Hindawi}

Submit your manuscripts at

http://www.hindawi.com
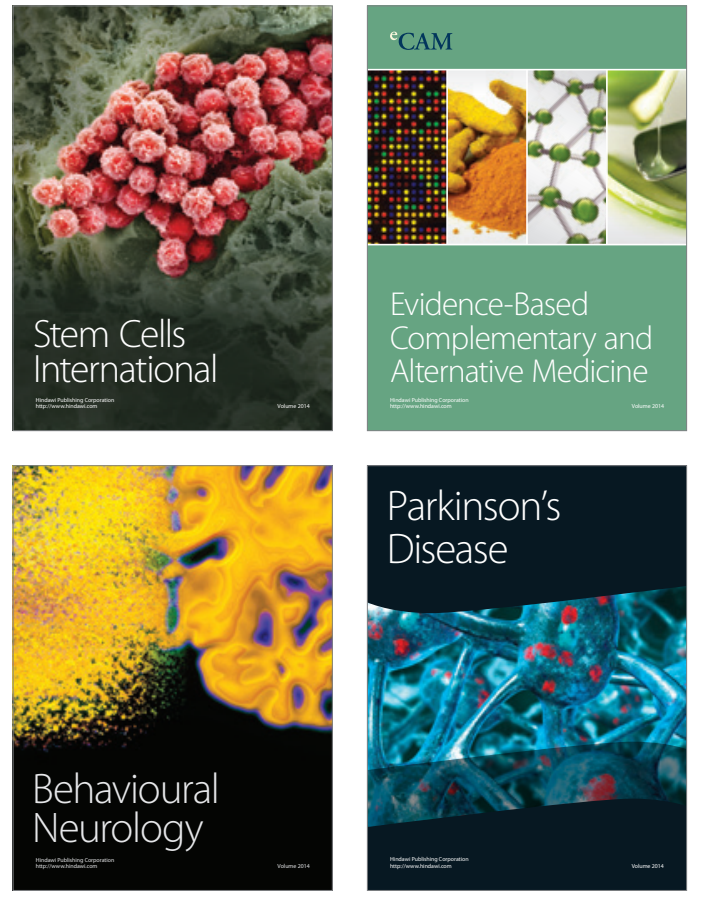
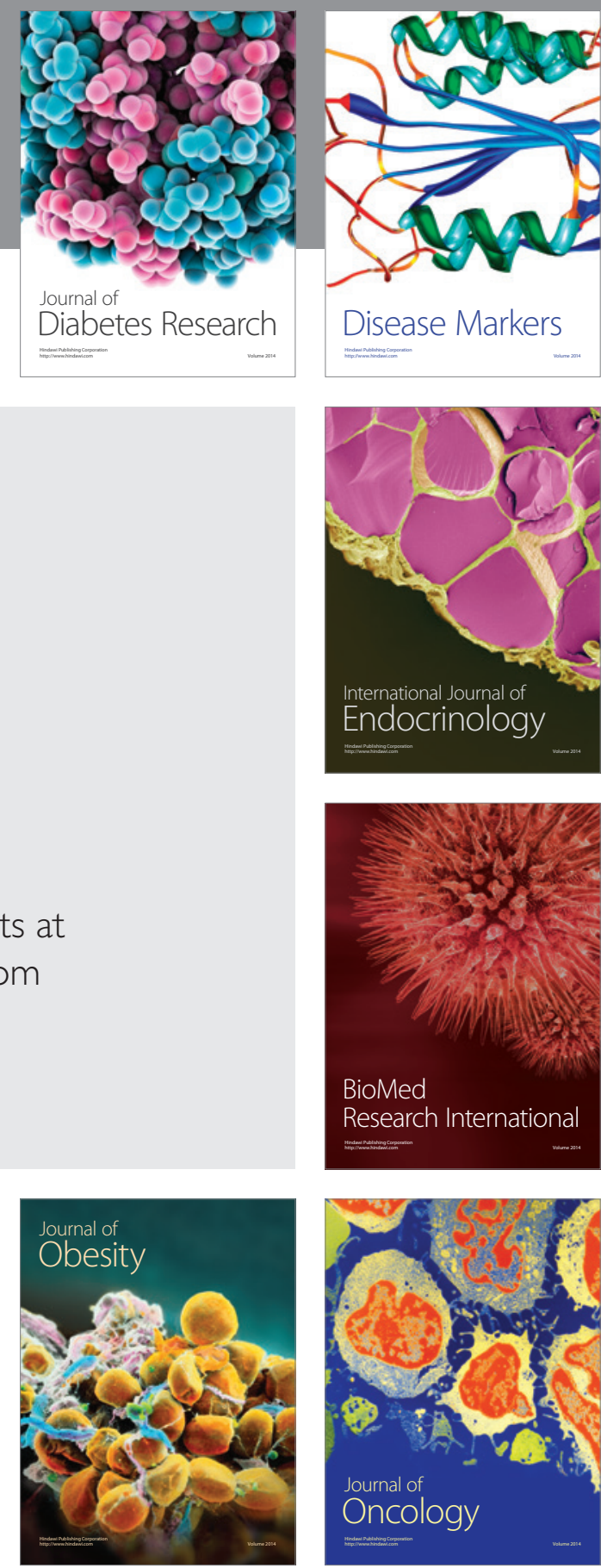

Disease Markers
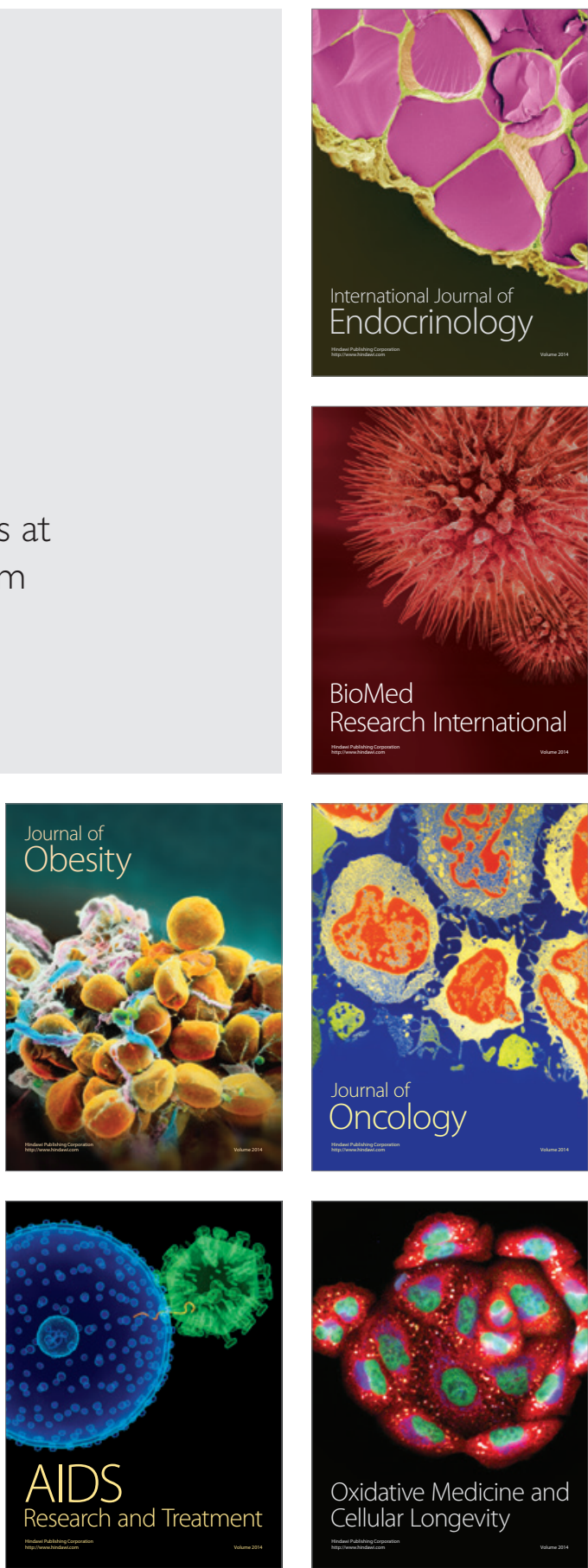